\title{
DESORDENS MÚSCULO-ESQUELÉTICAS EM ADOLESCENTES TRABALHADORES
}

\section{MUSCULOSKELETAL DISORDERS IN ADOLESCENT WORKERS}

\author{
Vanusa Caiafa Caetano * \\ Luiz Cláudio Ribeiro ** \\ Danielle Teles da Cruz *** \\ Carmen Ildes Rodrigues Fróes Asmus ****
}

Caetano VC et al. Desordens Músculo-Esqueléticas em Adolescentes Trabalhadores. Rev Bras Crescimento Desenvolvimento Hum. 2008; 18(3): 264-274

\section{Resumo:}

O trabalho infantil é um fenômeno mundial de grandes proporções, especialmente em países subdesenvolvidos. Estima-se, em todo o mundo, que 352 milhões de crianças e adolescentes com idade inferior a 18 anos são economicamente ativos, incluindo trabalho remunerado, não-pago, ilegal e informal. Assim, o objetivo é descrever a prevalência de desordens no sistema músculo-esquelético em um grupo de adolescentes inseridos no Programa de Formação Profissionalizante na "Casa do Pequeno Jardineiro”, na cidade de Juiz de Fora, MG. O método constou de observação, análise e descrição das posturas adotadas durante o processo de trabalho, aplicação de um questionário de identificação das condições de vida e trabalho e a realização do teste de flexão em pé (TFP). A amostra total foi dividida em três grupos, avaliando a ocorrência de dor e/ou desconforto nos ombros, nos cotovelos, nos quadris ou coxas e nos pés, retração dos grupos musculares tríceps sural e isquiotibiais, presença de dor e/ou desconforto nos quadris ou coxas associado à retração dos grupos musculares tríceps sural e isquiotibiais. Em conclusão, os adolescentes submetidos a uma carga de trabalho elevada apresentaram maior freqüência de desordens no sistema músculo-esquelético.

Palavras-chave: Fisioterapia; adolescente trabalhador; desordens músculo-esqueléticas.

\footnotetext{
* Professora Assistente II do Departamento de Fisioterapia da Faculdade de Medicina da Universidade Federal de Juiz de Fora.

** $\quad$ Professor Adjunto IV do Departamento de Estatística da Universidade Federal de Juiz de Fora.

*** Fisioterapeuta. Departamento de Fisioterapia da Faculdade de Medicina da Universidade Federal de Juiz de Fora.

**** Professora Adjunta do Instituto de Estudos em Saúde Coletiva da Universidade Federal do Rio de Janeiro. Correspondência para: Vanusa Caiafa Caetano. UFJF/FACMED/Dept ${ }^{\circ}$ Fisioterapia - CCS - Bairro Martelos - Campus Universitário - Juiz de Fora, MG. CEP- 36036-330. - E-mail: vcaiafa@gmail.com
} 


\begin{abstract}
:
Labor in childhood is a world phenomenon of great proportions, especially in underdeveloped countries. It's estimated, around the world, that 352 million children and adolescents under the age of 18 years old are economically active, including remunerated and unremunerated, illegal and informal work. Thus, the aim is to describe the prevalence of musculoskeletal disorders in a group of adolescents inserted in a professional apprenticeship program at the "Casa do Pequeno Jardineiro” (House of the Little Gardener) in the city of Juiz de Fora, MG. The methodology used was observation; analysis and description of the postures used during the process of labor; administration of a questionnaire to identify the conditions of the lifestyle and work; and the flexion test in standing (FTS). The sample was divided into three groups, evaluating the presence of pain or discomfort on the shoulders, on the elbows, on the hips or thighs and on the feet, retraction of the hamstrings and calf muscles, and presence of pain and/or discomfort on the hips or thighs associated with the retraction of the hamstrings and calf muscles. In conclusion, the adolescents that were submitted to a higher load in work showed a higher frequency of disorders of the musculoskeletal system.
\end{abstract}

Key word: Physiotherapy; adolescent; work; musculoskeletal disorders.

\section{INTRODUÇÃO}

Durante o período da infância e da adolescência ocorre um processo dinâmico de maturação e crescimento, onde o indivíduo se desenvolve física, psicológica e culturalmente. Alguns danos, ocorridos nessas fases, muitas vezes não podem ser reparados, como aqueles resultantes de situações de risco nos ambientes de trabalho que propiciam a ocorrência de repercussões futuras sobre a saúde desta população.

O trabalho infantil é um fenômeno mundial de grandes proporções, especialmente em países subdesenvolvidos. Estima-se, em todo o mundo, que 352 milhões de crianças e adolescentes com idade inferior a 18 anos são economicamente ativos, incluindo trabalho remunerado, não-pago, ilegal e informal, segundo International Labour Organization (ILO). ${ }^{1}$

Nesta população, 88\% das crianças de 5 a 14 anos e $42 \%$ dos adolescentes de 15 a 17 anos trabalham em atividades inadequadas para a idade, que precisam ser eliminadas em res- peito a convenções e padrões internacionais estabelecidos pela Organização Internacional do Trabalho (OIT). No Brasil, a legislação vigente permite, sob proteção especial, o trabalho para maiores de 14 anos na condição de aprendiz e para maiores de 16 anos na condição de trabalhador, mas cerca de 9,3 milhões de crianças e adolescentes entre 10 e 17 anos trabalham. ${ }^{1,2}$ Entretanto, segundo dados do Instituto Brasileiro de Geografia e Estatística (IBGE) ${ }^{3}$, existem no Brasil cerca de 2 milhões de crianças entre 5 e 13 anos trabalhando. Sabese que também estão trabalhando 1 milhão e meio de adolescentes com 14 a 15 anos e 2 milhões com 16 a 17 anos. Entre estes, pouquíssimos poderiam ser incluídos na condição de aprendiz ou estão em situação de proteção especial. Utilizando-se estimativas internacionais, poder-se-ia inferir que pelo menos metade destes adolescentes trabalhadores estão inseridos em atividades perigosas. ${ }^{1}$

Muitas situações de trabalho e da vida cotidiana são prejudiciais à saúde e as doenças músculo-esqueletais constituem uma das mais 
importantes causas do absenteísmo e da incapacitação para o trabalho. ${ }^{4,5}$ Estas lesões podem ser atribuídas ao trabalho repetitivo ou com sobrecarga, a fatores ambientais e/ou organizacionais, ao uso inadequado de equipamentos e a tarefas associadas à pressão psíquica no ambiente de trabalho.

Desordens e sintomas álgicos são comuns na população trabalhadora, e ocorrem predominantemente na região lombar, pescoço e membros superiores. De etiologia multifatorial, as síndromes dolorosas músculo-esqueléticas são freqüentemente relatadas, nos 6 a 12 meses iniciais de jornadas laborais. A sintomatologia pode aparecer em fases precoces da vida, infância ou adolescência, depois de moderada exposição a diversos fatores ou condições ambientais. ${ }^{6}$

Pouca atenção tem sido dada aos adultos que foram expostos a condições inadequadas de trabalho quando crianças. Neste sentido, não se conhece muito sobre a cascata de eventos que resultam em efeitos adversos no sistema músculo-esquelético. ${ }^{7}$ Assim, o objetivo é pesquisar a prevalência de desordens no sistema músculo-esquelético num grupo de adolescentes inseridos em um curso profissionalizante de jardinagem.

\section{MÉTODO}

Este estudo foi realizado em um grupo de 58 adolescentes, inseridos no Programa de Formação Profissionalizante da Casa do Pequeno Jardineiro (CPJ) da Associação Municipal de Assistência à Comunidade (AMAC), na cidade de Juiz de Fora, MG. A amostra foi definida obedecendo ao seguinte critério de inclusão: todos os adolescentes participantes e freqüentes no programa supracitado. Não foi excluído nenhum adolescente, uma vez que no momento da coleta dos dados todos estavam presentes. A coleta dos dados foi realizada em duas etapas.
Na primeira etapa foram observadas as posturas utilizadas durante atividade laboral, através de visitas exploratórias de observação e acompanhamento no local durante cinco dias, em período integral. A análise do material coletado seguiu as seguintes modalidades práticas da observação: deslocamentos da coluna vertebral, tronco, membros superiores e inferiores. A cada uma dessas classes observáveis foram associadas questões técnicas relativas ao registro, hipóteses específicas e à fidelidade das descrições das atividades, conforme preconizado por Guérin et al (2001). ${ }^{8}$

Subsequentemente, a segunda etapa foi realizada durante quatro dias consecutivos em período integral e constou da aplicação de uma entrevista estruturada e realização do teste físico, ambos aplicados em todo o grupo, individualmente. A entrevista foi elaborada com base no questionário aplicado por Asmus e Câmara (2004) $)^{9}$ e no Standardised Nordic questionnaires for the analysis of musculoskeletal symptoms ${ }^{10}$, validado em 2002, por Pinheiro, Tróccolia e Carvalho. ${ }^{11}$ Apresentou os seguintes blocos: identificação, dados demográficos, história ocupacional, história social (renda familiar, moradia, saneamento básico), morbidade específica (anamnese dirigida para sistema músculo-esqueletal), avaliação clínica (história clínica, história familiar, hábitos), exame físico (coluna vertebral e aparelho osteo-mioarticular) e teste de flexibilidade.

O teste de flexibilidade constou do Teste de Flexão de Pé(TFP), citado por Busquet (2001) ${ }^{12}$, tendo como objetivo observar uma cifose harmoniosa da região occipital à pélvica, devendo continuar no nível dos membros inferiores por uma extensão dos joelhos, verificando possíveis encurtamentos e/ou retrações musculares (tríceps sural, isquiotibiais, quadrado lombar).

Os adolescentes foram classificados em três grupos: 
Grupo 1: adolescentes que realizavam trabalho adicional concomitante ao curso profissionalizante (17 adolescentes); Grupo 2: adolescentes que haviam trabalhado antes do curso profissionalizante (52 adolescentes); Grupo 3: adolescentes que nunca realizaram outra atividade laboral antes do curso profissionalizante da CPJ (6 adolescentes), descritos na Figura 1.

Os dados obtidos dos questionários estruturados foram digitados e analisados no programa Statistical Package for the Social Sciences (SPSS) e a significância das diferenças entre as frequências dos grupos foi

\section{Figura 1: História Ocupacional dos adolescentes estudados, atual e pregressa}

ATUAL

Adolescentes que não realizavam outro tipo de trabalho: 41 (70,7\%).Adolescentes que realizavam outro tipo de trabalho: 17 (29,3\%).Outras atividades que os adolescentes realizam:- jardinagem, capina: 6 ( 33,3\%).- servente de obra: 4 (22,3\%).- auxiliar de mecânico, lavador de carros: 2 (11,1\%) em cada atividade.- pintura em geral, montagem de festas, estamparia industrial: 1 (5,6\%) em cada atividade.

Tempo de trabalho dos adolescentes que realizam ou já realizaram outro tipo de trabalho:

- > de 2 anos: 30 (51,7\%).

- Pelo menos 2 anos: 22 (37,9\%).

- Nunca trabalharam antes do curso profissionalizante: 6 (10,3\%).

\section{PREGRESSA}

Adolescentes que já realizaram outro tipo de trabalho: 52 (89,7\%).Atividades já realizadas:- servente de obra: 19(36,5\%).- horticultura, jardinagem, capina, limpeza de terrenos, corte de eucaliptos: 10 (19,2\%).chapa de caminhão: 6 (11,5\%).- auxiliar de mecânico 5 (9,6\%).- vendedor ambulante 2 (5,8\%).- pinturas em geral, marcenaria: 2 (3,8\%) em cada atividade.empacotador industrial, lavador de carros, serviços domésticos, agropecuária, arrematador industrial: 1 (1,9\%) em cada atividade.

Média da idade de início das atividades laborais de todos os adolescentes que realizavam ou já realizaram:

- Aos 16 anos: 04 (7,7\%).

- Aos 15 anos: 20 (38,5\%).

- Aos 14 anos: 17 (32,7\%).

- Abaixo de 14 anos: 11 (21,1\%). verificada por meio do Teste Exato de Fisher. A utilização deste teste permitiu a investigação das possíveis relações entre retrações musculares, presença de cansaço, dor e/ou desconforto no aparelho locomotor e alterações no aparato osteomioarticular entre os grupos de adolescentes estudados.

A opção por este teste deve-se ao fato de que a amostra é relativamente pequena, o que ocasiona freqüências esperadas menores do que cinco quando do cruzamento das variáveis. O nível de significância adotado foi de 5\%.

Toda a metodologia foi desenvolvida obedecendo aos princípios éticos da Resolução 196/96 do Conselho Nacional de Saúde e o estudo foi aprovado pelo Comitê de Ética do Instituto de Estudos de Saúde Coletiva da Uni- versidade Federal do Rio de Janeiro, sob o parecer 48/2006 e número do processo 05/2006.

\section{RESULTADOS}

Todos os 58 adolescentes entrevistados eram do sexo masculino, sendo que 48 estavam com 16 anos (82,8\%) e $10(17,2 \%)$ com idade entre 14 e 15 anos. Todos freqüentavam a escola, 55 (94,8\%) estavam cursando o ensino fundamental e 3 (5,2\%) o ensino médio. Esse programa tem a duração de quatro meses, envolvendo atividades teórico-práticas e estágio supervisionado em praças, jardins da comunidade e empresas conveniadas, com carga horária de 4 horas por dia, dividido em uma turma matutina e outra vespertina. 
Em todas as moradias dos adolescentes entrevistados havia coleta de lixo, rede de esgoto e água instalada. Em $75 \%$ dos domicílios moravam entre 3 e 5 pessoas e em 25\% pelo menos 6 moradores. Nos 20 domicílios com até 4 moradores, 8 famílias (40,0\%) tinham renda menor que 1 salário mínimo e 12 (60,0\%) tinham renda entre 1 e 3 salários mínimos. Nos 38 domicílios com mais de 5 moradores, 15 (39,5\%) famílias tinham renda menor que 1 salário mínimo e 23 (60,5\%) tinham renda entre 1 e 3 salários mínimos.

Não tinham outras atividades de trabalho, além do curso profissionalizante, 41 (70,7\%) adolescentes e 17 (29,3\%) realizavam outros trabalhos adicionais. Na história ocupacional colhida, 52 adolescentes já haviam realizado algum tipo de trabalho anterior ao seu ingresso na CPJ, sendo que 11 relataram ter sofrido algum tipo de acidente de trabalho. Dentre os acidentes menciona- dos, foram encontrados 6 (54,5\%) referentes a cortes (facão, enxada, moto-serra, tesoura, vidro), $2(18,2 \%)$ a fraturas e traumas e os demais por queda de altura, queimadura e atropelamento (1 adolescente em cada situação). Os acidentes de trabalho relatados ocorreram em atividades laborais fora da CPJ.

A observação da atividade de trabalho revelou uma grande variedade de posturas estáticas e dinâmicas, combinadas com diferentes graus de movimentos dos segmentos corporais. As posturas representam um indicador complexo da atividade e dos constrangimentos que pesam sobre ela, sendo constituída pela organização relativa dos diferentes segmentos corporais. A combinação destes fatores desencadeia, freqüentemente, uma multiplicidade de posturas possíveis, portanto difíceis de codificar. ${ }^{8}$ A descrição da análise das posturas está na Figura 2 .

\section{Figura 2: Análise das posturas em adolescentes trabalhadores}

Posturas de trabalho mais observadas durante nosso estudo:

- sentadas, agachadas, em pé.

- tempo variável em relação aos movimentos dos membros superiores e rotação de tronco (posturas dinâmicas).

- Predomínio da postura sentada com flexão anterior de tronco, pescoço e cabeça (postura estática).

Movimentos de membros inferiores:

- agachados, com predomínio da flexão de joelhos e coxo femoral.

- flexão extensão de joelho

- dorsiflexão e flexão plantar.
Movimentos de membros superiores:

- repetitivos movimentos de flexão e extensão: cotovelos, ombros, punhos e dedos.

- abdução e adução horizontal de ombros.

- oponência do polegar.

Movimentos de tronco: manutenção prolongada da flexão anterior, coluna cervical e lombar.

- movimentos rotacionais de tronco.
Em relação à ocorrência de dor e/ou desconforto em um ou mais segmentos, 29 (50\%) adolescentes entrevistados referiram dor e/ou desconforto na região lombar, 19 (32,8\%) nos joelhos, $14(24,1 \%)$ nas costas e pescoço, 11 (19\%) nos quadris ou coxas e $5(8,6 \%)$ nos ombros.

Quando questionados acerca da existência de sintomas no período retroativo de sete dias, $25(43,1 \%)$ adolescentes referiram sintomatologia dolorosa e/ou desconforto nos órgãos locomotores, 37 (63,8\%) referiram sensação de cansaço no corpo, 8 (13,8\%) impossibilidade de realizar suas atividades devido ao quadro álgico, e 3 (6,0\%) relataram terem sido avaliados por um profissional de saúde, nos últimos 12 meses, devido a esta sintomatologia. 
No exame postural ortostático, encontrou-se alteração no aparelho osteomioarticular em 22 (38\%) adolescentes. A alteração mais freqüente foi hiperlordose lombar em 8 $(13,8 \%)$, seguida pela escoliose toracolombar em $5(8,6 \%)$, geno-varo em 5 (8,6\%), e hipercifose em 4 (6,9\%) adolescentes. Os demais não apresentaram alterações no aparelho osteomioarticular.

Verificou-se maior retração dos músculos tríceps sural, isquiotibiais e quadrado lombar. Os dados encontrados de retrações dos músculos testados são resumidos na Tabela 1.

Foi observada diferença estatisticamente significativa entre os adolescentes do grupo $1 \mathrm{e}$ do grupo 3, quando referenciada a ocorrência de dor e/ou desconforto nos ombros ( $\mathrm{p}=0,009)$, nos cotovelos $(\mathrm{p}=0,025)$, nos quadris ou coxas ( $p=0,005)$ e nos pés $(\mathrm{p}=0,022)$. Encontrou-se também uma diferença estatisticamente significativa quanto à retração dos grupos musculares tríceps sural e isquiotibiais ( $p=0,012)$, entre os adolescentes do grupo 1 com relação aos adolescentes dos grupos 2 e 3 . A presença de dor e/ou desconforto nos quadris ou coxas associado à retração dos grupos musculares tríceps sural e isquiotibiais nos mostraram uma diferença estatisticamente significativa ( $p=0,010)$, nos adolescentes do grupo 1 com relação aos dos grupos 2 e 3 .

$\mathrm{O}$ cansaço associado à retração muscular de tríceps sural dos adolescentes do grupo 1 foi estatisticamente significativo $(p=0,044)$, assim como a retração muscular de tríceps sural e isquiotibiais associados ao tempo de atividade laboral $(\mathrm{p}=0,049)$.

Não foi encontrada significância estatística nos adolescentes que realizavam outro tipo de trabalho nas seguintes situações: dor e/ou desconforto nas costas ( $\mathrm{p}=0,62)$ e nos joelhos $(\mathrm{p}=1,00)$ associados à retração dos músculos tríceps sural e isquiotibiais; dor e/ou desconforto no pescoço e retração de tríceps sural ( $\mathrm{p}=0,096)$ e cansaço e retração do tríceps sural $(\mathrm{p}=0,059)$.

Todos os adolescentes receberam orientações posturais preventivas quanto ao uso inadequado do corpo nas atividades da vida diária e laborais desenvolvidas na CPJ. Aqueles identificados com alterações, dor e/ou desconforto foram encaminhados ao Hospital Universitário da Universidade Federal de Juiz de Fora, para assistência médica e fisioterapêutica.

\section{DISCUSSÃO}

O adolescente, diferentemente do adulto, requer cuidados e várias considerações sobre a sua inclusão no mercado de trabalho, especialmente quando esta ocorre precocemente. As pesquisas e a realidade têm demonstrado que a pobreza é a causa básica que força as crianças e os adolescentes a entrarem para o mercado de trabalho. ${ }^{13}$

As atividades desenvolvidas pelos adolescentes no curso profissionalizante de jardi-

Tabela 1: Freqüência de retração muscular de acordo com o Teste de Flexão de Pé

\begin{tabular}{cc}
\hline Músculos & Freqüência de retração muscular \\
\hline Tríceps sural & $9(15,5 \%)$ \\
Tríceps sural + ísquios tibiais & $13(22,4 \%)$ \\
Tríceps sural + quadrado lombar & $13(22,4 \%)$ \\
Tríceps sural + ísquios tibiais + quadrado lombar & $23(39,7 \%)$ \\
Total & $58(100 \%)$ \\
\hline
\end{tabular}


nagem, especialmente quando associadas a atividade de trabalho adicionais, podem contribuir de maneira cumulativa como fatores de risco para as desordens músculo-esqueléticas encontradas no presente estudo.

Ressaltamos que, a expressão "desordens” é mais abrangente que "lesão”, onde alterações do equilíbrio funcional e estrutural dos locais acometidos estão presentes, desde o início de suas manifestações, representadas como fadiga, desconforto e sensação de peso, enquanto que as lesões podem ser identificadas nos estágios mais tardios do processo. ${ }^{14}$

A demanda calórica, inerente à execução das atividades laborais, desvia as reservas energéticas do adolescente, impedindo o desenvolvimento de massa corporal e propiciando fadiga secundária. ${ }^{9}$ Grandjean (1998) ${ }^{15}$ afirma que o estado de fadiga está relacionado a uma sensação subjetiva de cansaço, referida como sensação de peso e indolência, ausência de motivação para o trabalho físico e mental. Sendo o cansaço um mecanismo de proteção a maiores sobrecargas, para que os processos normais de restabelecimento possam ocorrer no momento de repouso.

O expressivo número de adolescentes entrevistados que relataram sentir cansaço, independentemente se realizavam outra atividade laboral, pode ser devido às diferentes respostas individuais à sobrecarga laboral e/ou atividade escolar concomitante. As consequências de uma mesma causa externa nem sempre afetam da mesma maneira ou com a mesma intensidade todos os indivíduos que a ela são submetidos. ${ }^{8}$

O processo de trabalho na CPJ era diversificado e abrangente. Os adolescentes realizavam diferentes atividades diárias, assim como várias posturas corporais de acordo com a atividade de aprendizagem. No ambiente de trabalho (a céu aberto) os adolescentes ficavam expostos ao sol, frio, garoa, umidade relativa do ar, poeira, terra (manuseio com as mãos sem luvas). E as ferramentas utilizadas observadas foram: enxada, tesoura, carrinho de mão, pá, garfo, vassoura (folhas de árvore).

O processo de trabalho nas atividades de jardinagem envolvia simultaneamente contrações musculares estáticas e dinâmicas, durante os movimentos intermitentes de levantar, agachar e empurrar, rotações e inclinações laterais com o tronco, movimentos repetitivos de membros superiores e inferiores e manutenção prolongada de posturas.

Certas atividades ocupacionais podem exigir posturas que impõem tensões contínuas a determinados grupos musculares, como o caso do trabalho em atividades de jardinagem do presente estudo. Dentre os músculos mais envolvidos no desencadeamento da lombalgia estão os eretores da coluna, posteriores da coxa e quadrado lombar. ${ }^{16}$

O fato de trabalhar em uma postura estática faz com que diversos músculos sejam acionados, permanecendo em estado de tensão constante, tanto para sustentar o corpo contra a gravidade ou para manter uma determinada posição articular. O trabalho estático, conforme define Ragasson (2004) ${ }^{17}$, é aquele que exige contração contínua de alguns músculos, sendo altamente fatigante e sempre que possível deve ser evitado. Este pode contribuir para o surgimento de desgastes articulares, dos discos intervertebrais e tendões ${ }^{15}$, tendo ainda como resposta alterações circulatórias e metabólicas locais, tornandoas auto-perpetuantes. ${ }^{18}$

A atividade do plantio de sementes em saquinhos, estando o adolescente sentado em um toquinho de madeira, promove um trabalho predominantemente estático de tronco, lombar, cervical, ombros e membros inferiores. Concomitantemente suportam a flexão e extensão de cotovelos sem apoio, ao mesmo tempo em que punhos e dedos executam um trabalho principalmente dinâmico. Já que o trabalho estático é mais cansativo que o dinâmico, este se torna o componente de maior relevância. ${ }^{15} \mathrm{O}$ 
trabalho prolongado nesta postura, no plantio de sementes observado no presente estudo, poderá contribuir com sintomas de fadiga e algias no sistema vertebral, ombros e cotovelos.

Isso pode explicar o fato do grupo 1 , adolescentes submetidos a uma maior sobrecarga, terem apresentado maior relato de dor e/ou desconforto nos ombros ( $\mathrm{p}=0,009)$, nos cotovelos ( $p=0,0025)$, nos quadris ou coxas $(\mathrm{p}=0,005)$ e nos pés $(\mathrm{p}=0,022)$. Juntamente com essa maior sobrecarga imposta pelo trabalho estático, há também um maior consumo de energia e necessidade de períodos mais prolongados para recuperação, o que não é possível para esse grupo.

Todos os músculos testados apresentaram retração, o que resulta em diminuição da amplitude do movimento articular e da flexibilidade. Cabe ressaltar que a diminuição da flexibilidade é um achado comum entre meninos de 10 a 18 anos de idade, conforme Alter (1996)..$^{19}$

Segundo Corbin e Noble $(1980)^{20}$, ao se avaliar a flexibilidade de crianças e adolescentes deve ser considerada a fase do crescimento. Durante períodos de crescimento rápido, os ossos crescem mais do que os músculos se alongam, resultando em aumento de tensão musculotendinosa sobre uma articulação, e consequentemente redução da flexibilidade. ${ }^{19}$

Haskell, Montoye e Orestein (1985) ${ }^{21}$, consideram que uma boa flexibilidade na região lombar, membros inferiores e ombros podem contribuir potencialmente na redução de lesões, desconfortos e algias, favorecendo o desempenho nas atividades da vida diária.

Salvador, Neto e Ferrari $(2005)^{16}$, revelaram que atividades laborais podem contribuir para a dor lombar em trabalhadores, sendo o principal fator desencadeante desse quadro álgico. Wright, Barrow e Fisher $(1995)^{22}$, afirmaram que a atividade vigorosa desempenhada em uma determinada função está correlacionada com a dor músculo- esquelética na coluna lombar em trabalhadores de 18 a 39 anos.

Os adolescentes do grupo 1, apresentaram maior dor e/ou desconforto nos quadris/ coxas associado à retração de tríceps sural e isquiotibiais ( $p=0,010)$, quando comparados com os grupos 2 e 3. Dessa forma, podemos inferir que esses adolescentes podem estar propícios a desenvolver futuramente quadros de dor e/ou desconforto articular na região lombo-pélvica.

O estudo realizado por Carazzato, Cabrita e Castropil (1996) ${ }^{23}$, determinou a incidência de lesões no aparelho locomotor em praticantes de judô de alto nível. Verificou-se maior predomínio de lesões naqueles que iniciaram seus treinamentos em idade mais precoce e que permaneceram em atividade competitiva por maior tempo. O que nos sugere a relação existente entre a demanda imposta pelas atividades e a capacidade física individual dos sujeitos.

No estudo de Harraby et al (1999) ${ }^{24}$, a dor lombar em adolescentes é um problema comum que aumenta com a idade, o que pode representar um risco de recorrências na fase adulta. Os adolescentes deste estudo apresentaram uma demanda imposta na capacidade física provenientes de competições esportivas, atividades laborativas e escolares, que geraram sobrecargas mecânicas no sistema músculoesquelético. O que corrobora com os achados do grupo1 do nosso estudo.

Não encontramos significância estatística nos adolescentes do grupo 1, nas situações de: dor e/ou desconforto no pescoço associado à retração de tríceps sural $(\mathrm{p}=0,096)$; dor e/ou desconforto nos joelhos associado à retração de tríceps sural e isquiotibiais $(\mathrm{p}=1,00)$; cansaço associado à retração do tríceps sural ( $\mathrm{p}=0,059)$; dor e/ou desconforto nas costas associada à retração de tríceps sural e isquiotibiais $(p=0,062)$. Os dois últimos dados sugerem uma diferença marginalmente significativa, mas não são su- 
ficientemente fortes para garantirmos a associação entre os fatores.

Apesar dos resultados não apresentarem significância estatística, podemos sugerir uma possível relação da dor e/ou desconforto nas costas e joelhos com a retração de tríceps sural e isquiotibiais. A retração destes músculos leva a uma sobrecarga mecânica nas articulações dos membros inferiores, principalmente quando se adota flexão anterior de tronco ${ }^{19}$, postura esta predominante nas atividades de jardinagem. A sobrecarga mecânica articular pode estar também relacionada com a sensação subjetiva de cansaço. Mannion e Dolan (1994) $)^{25}$ observaram que a fadiga nos membros inferiores pode interferir nos testes de resistência isométrica dos eretores da coluna vertebral.

A presença de dor no sistema músculo-esquelético pode ser um indicativo de sofrimento, mesmo quando não identificadas alterações nos exames clínicos complementares. ${ }^{9}$ O crescimento e o desenvolvimento na adolescência são definidos pela interação entre o potencial genético e as condições ambientais, sendo de fundamental importância a compreensão dos efeitos da inserção precoce no mercado de trabalho, uma vez que $20 \%$ da altura e $60 \%$ da maturidade óssea que o indivíduo terá quando adulto, correspondem ao desenvolvimento somático na fase da adolescência. ${ }^{26}$

O presente estudo visou a identificar desordens no sistema músculo-esquelético,

\section{REFERÊNCIAS}

1. ILO (International Labour

Organization). Every childs counts:

new global estimates on child labour.

ILO, Geneva, 2002.

2. ILO (International Labour

Organization). Statistics on working children and hazardous child labour in brief. ILO, Geneva, 1998.

3. Instituto Brasileiro de Geografia e Estatística (IBGE). Pesquisa Nacional decorrentes do trabalho precoce. A avaliação fisioterapêutica permitiu, em nível individual, o diagnóstico precoce dos danos e alterações no sistema músculo-esquelético. Em nível coletivo foram identificadas as condições de saúde da população estudada e seus determinantes ocupacionais. Conseqüentemente, permitiu também propor as mudanças das condições de trabalho requeridas para minimizar os riscos para a saúde dos adolescentes envolvidos.

Os adolescentes submetidos a uma maior carga de trabalho apresentaram uma maior freqüência de desordens no sistema músculo-esquelético. A demanda imposta a esse sistema possui uma relação direta com a capacidade física, o período da adolescência e atividades laborais. Assim, torna-se necessário fornecer subsídios quanto à prevenção e orientação das atividades que determinam essas desordens.

Os cuidados com a saúde do jovem trabalhador são necessários e, muitas vezes, complexos frente aos determinantes físicos, psíquicos, econômicos e socioculturais. O trabalho precoce representa um problema de extrema relevância no contexto da Saúde Pública do Brasil, sendo necessário haver políticas públicas compensatórias para propiciar ao indivíduo oportunidade, crescimento e desenvolvimento na aplicação da legislação que regula o trabalho do menor.

por Amostra de Domicílios: Trabalho Infantil 2001. Rio de Janeiro: 2003.

4. Ribeiro HP. Lesões por esforços repetitivos (LER): uma doença emblemática. Cad. Saúde Pública. 1997;13(2):85-93.

5. Cherem AJ. A prevenção do Pathos: uma proposta de protocolo para diagnóstico dos distúrbios osteomusculares relacionados ao trabalho [dissertação]. Florianópolis: Pós-Graduação de Engenharia de Produção e Sistemas da 
Universidade Federal de Santa Catarina; 1998.

6. John D, Macarthur CT. Muscle Tension. Research Network on Socioeconomic Status and Health. [acesso em 2006 fev 10]; Disponível em: http:// www.macses.ucsf.edu/research/ Ailostatic/notebook/muscle.html

7. Selevan SG, Kimmel CA, Mendola P. Identifying critical windows of exposure for children health. Environ Health Perspect. 2000 Jun;108(supl 3):451-55.

8. Guérin F, Laville A, Daniellou F, Duraffourg J, Kerguelen A. Compreender o trabalho para transformá-lo. A prática da ergonomia. São Paulo: Editora Edgard Blücher Ltda; 2001.

9. Asmus CIRF, Câmara VM. Saúde de adolescentes trabalhadores em garimpos de diamantes. Cad. Saúde Coletiva. 2004;12(2):179-96.

10. Kuorinka I, Jonsson B, Kilbom A, Vinterberg N, Beiring-Sorensen F, Andersson G, et al. Standardised Nordic questionnaires for the analysis of muculoskeletal symptoms. Applied Ergonomics. 1987 Sept;18(3):233-37.

11. Pinheiro FA, Tróccolia BT, Carvalho CV. Validação do questionário Nórdico de sintomas osteomusculares como medida de morbidade. Rev Saúde Pública. 2002;36(3):307-12.

12. Busquet L. Tratamento pelas cadeias musculares. In: As cadeias musculares. $1^{a}$ ed. São Paulo: Edições Busquet; 2001. p. 115-20.

13. Sato, L. LER: objeto e pretexto para a construção do campo trabalho e saúde. Cad. Saúde Pública. 2001;17(1):147-52.

14. Batista EB, Borges FD, Dias LP, Fabris G, Frigeri F, Salmasco C. Lesões por esforços repetitivos em digitadores do Centro de Processamento de dados do
Banestado, Londrina, Paraná. Rev. bras. fisioter. 1997;4(2):83-91.

15. Grandjean E. Manual de ergonomia: adaptando o trabalho ao homem. Porto Alegre: Artes Médicas; 1998.

16. Salvador D, Neto PED, Ferrari, FP. Aplicação de técnica de energia muscular em coletores de lixo com lombalgia mecânica aguda. Fisioterapia e Pesquisa, 2005;12(2):20-7.

17. Ragasson CAP. Qualidade no trabalho: estudo das condições de trabalho. Cascavel: Editora Coluna do Saber; 2004.

18. Zapater AR, Silveira DM, Vitta A, Padovani CR, Silva JCP. Postura sentada: a eficácia de um programa de educação para escolares. Ciência \& Saúde Coletiva, 2004;9(1):191-99.

19. Alter MJ. Ciência da flexibilidade. Porto Alegre: Editora Artes Médicas Sul Ltda; 1996.

20. Corbin CB, Noble L. Flexibility: A major component of physical fitness. Journal of Physical Education and Recreation. 1980;51(6):23-60.

21. Haskell WL, Montoye, HJ, Orestein, D. Physical activity and exercise to achieve health-realted fitness components. Public Health Rep. 1985;100:202-12.

22. Wright, D, Barrow S, Fisher AD. Influence of psychological and behavioral factors on consultation for back pain. Br J Rheumatol. 1995;34:156-61.

23. Carazzato JG, Cabrita H, Castropil W. Repercussão no aparelho locomotor da prática de judô de alto nível. Rev Bras Ortop. 1996;31(12):957-68.

24. Harreby M, Nygaard B, Jessen T, Larsen E, Storr-Paulsen A, Lindahl A, et al. Risk factors low back pain in a cohort of 1389 Danish school children: an epidemiologic study. Eur Spine J. 1999;8:444-50. 
25. Mannion AF, Dolan P.

Electromyographic median frequency changes during isometric contraction of the back extensors to fatigue. Spine. 1994;19(11):1223-9.
26. Martins IS, Fischer FM, Oliveira DB, Teixeira LR, Costa LAR, Marinho SP, et al. Crescimento e trabalho de estudantes de ensino fundamental e médio em São Paulo, Brasill. Rev Saúde Pública. 2002;36(1):19-25.

Recebido em: 10/08/2007 Reapresentado: 28/06/2008 Modificado em 08/11/2008 Aceito em: 20/12/2008 